\title{
Understandable and not surprising
}

\section{To the Editor:}

With regard to having patients drink soda water to improve quality of myocardial scintigraphy images, ${ }^{1}$ several key points are worth noting. The authors demonstrated that swallowing a small amount of an effervescent liquid improved image quality of the inferior wall and helped to diminish image degradation due to intestinal activity. They contended that the method is "effective, simple, and inexpensive" and "will be a useful alternative to other methods." I agree.

However, the use of an oral effervescent agent to minimize intestinal activity and to decrease inferior (sometimes termed "diaphragmatic") attenuation artifact in myocardial scintigraphy had been previously described. $^{2}$ Also, the use of an imbibed liquid effervescent agent for the same purposes has been granted a US patent (retroactive to submissions of 2003), ${ }^{3}$ which included (a) myocardial SPECT scintigraphy done with the introduction of carbon dioxide gas into the stomach and (b) such imaging utilizing an imbibed liquid containing dissolved gas. The authors also mentioned the benefit at rest acquisition in addition to stress; this likewise had previously been discussed. ${ }^{2}$

The authors did not mention prior elucidation that inferior artifact is due to attenuation by thick stomach wall (rather than diaphragm), which readily explains why the effervescent liquid they administered worked. It distended the stomach and thinned its wall, and filled the upper portion of the stomach (closest to inferior myocardium) with gas, creating a non-attenuating scintigraphic "imaging window. $, 2,3$
One can readily envision why what the authors called "the Monzen position" could be of benefit in diminishing bowel activity. Were inferior attenuation artifact to be the primary culprit, their administration of effervescent liquid would be expected to work better in the upright, right lateral decubitus, or right lateral semiupright positions, as that should place more non attenuating gas near myocardium.

\author{
Samson Munn, MD, FACR \\ Department of Radiology \\ Tufts Medical Center \\ Boston, MA, USA \\ E-mail:02467@earthlink.net
}

\section{References}

1. Hara M, Monzen H, Futai R, Inagaki K, Shimoyama H, Morikawa $\mathrm{M}$, et al. Reduction of infracardiac intestinal activity by a small amount of soda water in technetium-99m tetrofosmin myocardial perfusion scintigraphy with adenosine stress. J Nucl Cardiol 2008; 15:241-5.

2. Munn S. The way to a man's heart is through his stomach: Much "diaphragmatic" attenuation is likely gastric, and effervescent granules enhance cardiac imaging. Eur J Radiol 2004;52:271-5.

3. Munn CS. Enhanced cardiac radionuclide imaging techniques. United States Patent and Trademark Office 2009; patent \# 7,526,331. Cited therein are US provisional applications by the same author filed in 2003: 60/496,352, 60/489,738 and 60/488,541.

doi:10.1007/s12350-012-9647-1 